\title{
Niveles de metales pesados en muestras biológicas y su importancia en salud
}

\author{
Lesbia Rosa Tirado Amador*, Odon. , Farith Damián González-Martínez, \\ Odon. $_{1}$, Leonardo José Martínez Hernández, Est. Odon.., Luis Alberto \\ Wilches Vergara, Est. Odon.., José Nelson Celedón-Suárez, Est. Odon.
}

${ }_{1}$ Universidad de Cartagena, Cartagena, Colombia

Recibido: 27 de febrero del 2015. Aprobado: 14 de mayo del 2015.

*Autor de correspondencia: Lesbia Rosa Tirado Amador. Universidad de Cartagena, Campus Ciencias de la Salud, Facultad de Odontología, Departamento de investigaciones. Barrio Zaragocilla. Teléfono: (57) 5 6698172, ext: 115. Correo electrónico: Itiradoa@unicartagena.edu.co

Cómo citar este artículo: Tirado LR, González-Martínez FD, Martínez LJ, Wilches LA, Celedón-Suárez JN. Niveles de metales pesados en muestras biológicas y su importancia en salud. Rev Nac Odontol. 2015;11(21):83-99. doi: http://dx.doi. org/10.16925/od.v11i21.895

Resumen. En la sociedad del siglo Xxi —enfocada hacia el desarrollo socioeconómico-, la continua industrialización, la práctica de la minería y de actividades productivas como la agricultura resultan ser opciones atractivas; sin embargo, en el desarrollo de estas actividades, es frecuente el uso y la emisión de algunos químicos hacia el medio ambiente, entre los cuales se encuentran los metales pesados. En el organismo de los seres vivos, hay pequeñas cantidades de algunos metales pesados que pueden intervenir en reacciones bioquímicas, pero otros metales no representan utilidad biológica. Por esto, la exposición ocupacional y la exposición pasiva a metales pesados presentes en el ambiente o que provienen de la dieta deben ser reguladas, ya que cantidades superiores a las consideradas normales pueden ocasionar ciertas enfermedades. El control de la exposición a metales pesados incluye monitorear los niveles presentes en distintas matrices biológicas como la sangre, la orina, la saliva, el cabello y las uñas. Cada una de estas matrices estará indicada según el tipo de exposición que ha experimentado el individuo; además, ofrecerá algunas ventajas y limitaciones. Es por eso que la presente revisión pretende informar sobre los efectos ocasionados en el organismo por niveles excesivos de algunos metales pesados y la utilidad que ofrecen las matrices biológicas en la determinación de estos niveles.

Palabras clave: cabello, cádmio, cobre, mercurio, orina, plomo, saliva, sangre, uñas, zinc. 


\title{
Levels of Heavy Metals in Biological Samples and its Importance on Health
}

\begin{abstract}
In the society of the $21^{\text {st }}$ century - focused on the social economic developmentthe continuous industrialization, mining and productive activities such as agriculture, turn out to be attractive options. However, in the development of these activities, the use and emission of some chemical products to the environment, including heavy metals, is pretty frequent. Small amounts of some heavy metals may be in the organism of the living beings. Some of these heavy metals may intervene in biochemical reactions, while other metals do not have biological use. For this reason, occupational and passive exposure to heavy metals in the environment, or coming from the food, most be regulated, as quantities higher than the ones deemed normal may result in certain illnesses. Controlling exposures to heavy metals include monitoring the levels in different biological matrixes such as blood, urine, saliva, hair and nails. Each one of these matrixes shall be indicated according to the exposure experienced by the individual; it will also offer some advantages and limitations. That is why this revision aims to inform about the effects resulting from the excessive levels of some heavy metals, and the usefulness of biological matrixes to determine such levels.
\end{abstract}

Keywords: hair, cadmium, copper, mercury, urine, lead, saliva, blood, nails, zinc.

\section{Níveis de metais pesados em amostras biológicas e sua importância em saúde}

Resumo. Na sociedade do século XXI —-focada no desenvolvimento socioeconómico-, a continua industrialização, a prática da mineração e de atividades produtivas como a agricultura, são opções atraentes; no entanto, no desenvolvimento destas atividades é frequente o uso e a emissão de alguns químicos para o meio ambiente, dentro dos quais estão os metais pesados. No organismo dos seres vivos estão presentes pequenas quantidades de alguns metais pesados que podem intervir em reações bioquímicas; porém, outros metais não representam utilidade biológica. Por isso, a exposição ocupacional e a exposição passiva a metais pesados presentes no ambiente ou que provem da dieta deve ser regulada, porque em quantidades superiores às consideradas normais podem ocasionar algumas doenças. O controle da exposição a metais pesados inclui monitorar os níveis presentes em diferentes matrizes biológicas como o sangue, a urina, a saliva o cabelo e as unhas. Cada uma destas matrizes estará indicada de acordo com o tipo de exposição que tenha experimentado o indivíduo; além disso irá oferecer algumas vantagens e limitações. É por isso que esta revisão procura informar sobre os efeitos ocasionados no organismo por níveis excessivos de alguns metais pesados e a utilidade oferecida pelas matrizes biológicas na determinação de tais níveis.

Palavras-chave: cabelo, cadmio, cobre, mercúrio, urina, chumbo, saliva, sangue, unhas, zinco. 


\section{Introducción}

Las exposiciones ambientales constituyen un problema de salud pública en el mundo, por lo cual son necesarios estudios de monitoreo y control de la magnitud de estas exposiciones y sus efectos, con el fin de identificar el estado de salud en las poblaciones. Esto resulta útil para los sistemas de salud, debido a que los recursos que invierten se pueden redireccionar cada año hacia donde se requieren, teniendo en cuenta los principales riesgos de cada población.

En este sentido, es evidente que desde el enfoque ambiental, los individuos se exponen a diversas sustancias químicas que provienen principalmente de los desechos industriales, los residuos de actividades agrícolas, la combustión de hidrocarburos o la disposición inadecuada de residuos domésticos. Entre los diferentes elementos a los que se exponen las poblaciones se destacan los metales pesados, que son arrojados al ambiente como residuos de actividades mineras o industriales, pero también por su manipulación en actividades del sector automotriz, las fundiciones de metales industriales o artesanales, y la manipulación de combustibles [1].

La relevancia de monitorear los niveles de metales en diversas muestras biológicas radica en la posibilidad de evaluar sus variaciones por incremento, las cuales puedan ser sugestivas de exposiciones inadecuadas [1]. En la literatura se reportan diversas alteraciones sistémicas y deterioro en la salud de personas expuestas a altas concentraciones de metales pesados, ocasionando desde alteraciones locales en exposiciones directas, hasta afecciones secundarias a nivel del embrión, como consecuencia de una inadecuada exposición de la madre durante las etapas de gestación [1].

Con frecuencia, las mediciones de metales pesados en muestras biológicas se realizan mediante el uso de plasma sanguíneo como muestra de primera elección; sin embargo, en muchas ocasiones, se puede recurrir a muestras más fáciles de obtener y menos invasivas como el cabello, la orina, las uñas y la saliva [1]. En los últimos años, estas matrices para medición de metales pesados han llamado la atención de los investigadores, debido a que algunos sujetos de estudio que van a ser monitoreados utilizando sangre como matriz biológica suelen manifestar cierto grado de aprehensión, miedo y ansiedad cuando se trata de aceptar la punción venosa con aguja para obtener la muestra, por lo que otras matrices biológicas poco invasivas pueden convertirse en herramientas diagnósticas alternativas para evaluar las concentraciones de metales [1].

La presente revisión tiene como objetivo informar acerca de las funciones en el organismo de los metales pesados y la necesidad de monitorear los niveles en distintas muestras biológicas como sangre, saliva, cabello, orina y uñas, lo cual permitirá hacer asociaciones con la exposición (por dieta o por medio ambiente) y con alteraciones del metabolismo y excreción.

\section{Metales}

Se consideran metales un grupo de 70 elementos que tienen características comunes como el estado sólido en que se encuentran (a excepción del mercurio), sus elevados puntos de fusión y ebullición, solubilidad en agua, aspecto brillante, capacidad para generar sonidos, maleabilidad, termoconducción y electroconducción; además, producen óxidos de naturaleza básica. Los metales se han dividido según su densidad en metales ligeros, ultraligeros y metales pesados, a estos últimos pertenecen aquellos elementos cuya densidad es igual o superior a $5 \mathrm{~g} / \mathrm{cm}^{3}[2]$.

\section{Metales pesados}

A este grupo de metales pertenecen aquellos cuya densidad en forma elemental es igual o superior a 5 $\mathrm{g} / \mathrm{cm}^{3}$ o que tienen un número atómico mayor a 20 (excluyendo los metales alcalinos y alcalinotérreos) [2]. La presencia de estos elementos en la corteza terrestre no supera el $0,1 \%$. Los metales pesados se han agrupado en oligoelementos o micronutrientes, también denominados elementos traza, los cuales son necesarios en pequeñas cantidades para el organismo, ya que intervienen en algunas funciones bioquímicas; entre ellos están: arsénico (As), boro (B), cobalto $(\mathrm{Co})$, cromo $(\mathrm{Cr})$, cobre $(\mathrm{Cu})$, molibdeno $(\mathrm{Mo})$, manganeso $(\mathrm{Mn})$, níquel $(\mathrm{Ni})$, selenio (Se) y zinc (Zn).

El grupo de micronutrientes incluye elementos cuya función biológica es desconocida y son altamente tóxicos, aunque algunos pueden estar presentes en los seres vivos; a ellos pertenecen: 
bario $(\mathrm{Ba})$, cadmio $(\mathrm{Cd})$, mercurio $(\mathrm{Hg})$, plomo $(\mathrm{Pb})$, antimonio $(\mathrm{Sb})$ y bismuto $(\mathrm{Bi})[3]-[6]$.

La exposición excesiva a metales pesados genera efectos adversos que han sido documentados durante años, entre los cuales se puede mencionar, por ejemplo, que la exposición excesiva al cadmio (Cd) provoca daño renal y a nivel óseo ocasiona fracturas [7]. Sin embargo, la exposición a estos metales aumenta en algunas áreas, porque su uso y emisión ha incrementado como consecuencia de actividades industriales, entre otras; por ejemplo, desde mediados del siglo XIX se reportan emisiones de mercurio al medio ambiente, ya que este metal se utiliza en muchos países de América Latina para la minería de oro [8].

Por otro lado, es necesario considerar que, además de la frecuencia de exposición a metales pesados, es crucial el buen funcionamiento de los organismos en lo que respecta a los diferentes procesos para lograr la excreción de estas sustancias, lo cual evita que se generen niveles tóxicos, ya que estos metales tienden a bioacumularse $y$ experimentan biomagnificación, proceso en el que muchas toxinas alcanzan concentraciones perjudiciales en las células como consecuencia del recorrido por los diversos eslabones de las cadenas tróficas [9].

\section{Interés biológico de algunos metales pesados}

\section{Cobre $(\mathrm{Cu})$}

El cobre fue uno de los primeros metales utilizados por los seres humanos; su uso se remonta aproximadamente hacia el 5000 a. C. en la región del Egeo, donde fue empleado para la creación de objetos de arte valiosos [10]. La exposición de los seres humanos al cobre se debe principalmente al consumo de alimentos y agua potable, la ingesta de cobre en relación con los alimentos y el agua dependerá de la ubicación geográfica; generalmente, cerca de $20-25 \%$ de la ingesta de cobre proviene de agua potable [11].

Los déficits e incrementos excesivos del cobre han revestido gran importancia en la salud humana, debido a que es un elemento cuya deficiencia ha sido asociada con producción alterada de energía, anomalías en el metabolismo de la glucosa y el colesterol, incremento de iones de hierro, alteración estructural y fisiológica de la circulación sanguínea, afecciones cardiacas [12] y alteraciones a nivel de las células del sistema inmune [13], [14]. Otros efectos identificados son las alteraciones óseas en los niños con bajo peso al nacer [15]. En el aspecto genético, se ha reportado que la existencia de mutaciones a nivel de las proteínas de unión al cobre puede favorecer el desarrollo de diabetes [16], [17] y daño neurológico [18].

Por otra parte, la toxicidad ocasionada por este elemento se atribuye a la ingesta accidental [10]; la toxicidad aguda puede estar asociada con la existencia de estrés oxidativo en varias zonas del cuerpo o de alteraciones endocrinas [19]. Otros mecanismos reportados son exposiciones mediante la piel o las vías respiratorias [20]. La forma de toxicidad crónica surge como consecuencia de la exposición prolongada en pequeñas cantidades. En salud es posible mencionar la utilidad de emplear la medición de cobre por parte del odontólogo por medio de la saliva como matriz biológica, ya que es posible encontrar trazas de este elemento asociadas con caries dental, una enfermedad de gran importancia por su alta prevalencia y distribución a nivel mundial [21].

\section{Mercurio (Hg)}

El mercurio es un metal noble de color plateado que a temperatura ambiente se encuentra en estado líquido [22] y es soluble únicamente en soluciones oxidantes. Este metal puede formar soluciones llamadas amalgamas con otros elementos como oro, plata, platino, uranio, cobre, plomo, sodio y potasio [22], [23]. Tanto el mercurio como sus compuestos son muy tóxicos, por eso -y pese a que se ha utilizado desde siglos atrás en aplicaciones de medicina y odontología - existe la conjetura de que ciertas enfermedades pueden ser ocasionadas por la exposición indiscriminada a ciertos contaminantes que provocan aumento de los niveles de mercurio, por lo que se ha propagado la inquietud e incertidumbre pública sobre los posibles efectos indeseables asociados con las altas dosis de mercurio [22].

Existe gran variedad de fuentes ambientales de mercurio a las cuales se puede estar expuesto, incluidos desechos de las minas, efluentes industriales, agua de drenaje agrícola, embalses y la deposición atmosférica de generación de energía eléctrica [23]. Otra fuente de exposición es la dieta, tras consumir peces contaminados por metil-mercurio [24] o exponer a mercurio inorgánico $(\mathrm{Hg}$ 
elemental) en el medio bucal gracias a la presencia de restauraciones de uso odontológico [25].

En lo que respecta a exposición al mercurio por consumo de alimentos contaminados, existe gran preocupación por monitorear la exposición. Así algunas instituciones como la Agencia de Protección Ambiental de Estados Unidos y la Academia Nacional de Ciencias han establecido algunos valores de referencia permitidos, dentro de los cuales es posible mencionar que el mercurio en sangre y cabello no debe exceder 5,0 mg/L y $1,0 \mathrm{mg} / \mathrm{g}$, respectivamente. Otra consideración sugiere que el valor no sea superior a $0,1 \mathrm{mg} \mathrm{de} \mathrm{Hg} / \mathrm{kg}$ de peso corporal por día [26].

Como se mencionó, otra fuente de exposición al mercurio son las restauraciones de uso en odontología conocidas como amalgamas dentales, ya que su contenido incluye un $50 \%$ de mercurio. Por esta razón, algunos organismos han reconocido que existe la posibilidad de que se libere vapor mercurial a partir de estas restauraciones, aunque a muy bajos niveles, por lo que actualmente en el mundo se están desarrollando e implementando otras alternativas restauradoras en odontología para lograr reducir el uso de amalgamas dentales [27].

Lo anterior ratifica la gran variedad de fuentes de exposición al mercurio, un elemento metálico que no tiene aplicabilidad biológica, pues es considerado tóxico y perjudicial para la salud ya que induce efectos adversos en los sistemas nervioso e inmunitario, el aparato digestivo, la piel, los pulmones, los riñones y los ojos [25]. Entre estos efectos se incluye tos, dermatitis por contacto, dolor torácico, náuseas, vómitos, diarrea, aumento de la tensión arterial o la frecuencia cardiaca, sabor metálico en la boca, irritación ocular y cefaleas [26].

\section{Zinc (Zn)}

Este metal se aprecia como un elemento sólido de color gris a temperatura ambiente, es dúctil y maleable. Biológicamente, el zinc constituye un oligoelemento fundamental para la conformación y el funcionamiento del ácido desoxirribonucleico (ADN), enzimas, coenzimas y hormonas. Algunos autores como Florea [28] han reportado que el zinc se absorbe rápidamente y casi un $90 \%$ es almacenado; por tanto, la cantidad de zinc disponible en sangre puede variar con respecto a la cantidad real que hay en el organismo. Los sitios de depósito suelen ser el músculo esquelético, el encéfalo, los pulmones y el corazón.

La concentración varía muy poco con respecto a la ingesta de zinc, pero en el estudio de muestras biológicas como sangre o cabello las concentraciones varían en función de la cantidad ingerida [29].

Algunas fuentes de zinc en los alimentos son las carnes rojas, algunos mariscos como ostras, el germen de cereales y la leche [29]. Sin embargo, los requerimientos de zinc pueden variar a nivel mundial debido a factores ambientales; por ejemplo, en Reino Unido, se ha reportado que los hombres pueden requerir aproximadamente $7,3 \mathrm{mg}$ /día de zinc y las mujeres $5,5 \mathrm{mg} /$ día, mientras que en EE.UU. los reportes señalan valores de zinc hasta de $15 \mathrm{mg} /$ día para hombres y $12 \mathrm{mg} /$ día para mujeres [28].

Los rangos entre ingestas deficitarias y tóxicas de zinc parecen bastante amplios, lo que sugiere que el refuerzo de la dieta con zinc podría ser una sencilla solución a su baja disponibilidad. El principal efecto tóxico del zinc deriva del entorpecimiento del metabolismo normal de cobre, lo que conduce a una anemia en caso de nutrición parenteral o en personas con ingestas prolongadas superiores a $150 \mathrm{mg} /$ día [30].

Algunos autores han descrito que la deficiencia de zinc puede generar ciertas patologías como anemia, respuesta inmune tardía, déficit de hierro, hipogonadismo, enanismo, hepatoesplenomegalia [31], puesto que es un elemento relevante en el correcto funcionamiento en la bioquímica de los seres humanos, tanto así que ha sido utilizado con fines terapéuticos en diarreas agudas y crónicas, y en infecciones del tracto respiratorio inferior en lactantes y niños pequeños [32]. Por su parte, Prasad [33] considera que el zinc puede ayudar a la reducción de la duración y la intensidad de los síntomas del resfriado en un 50\%, especialmente la tos; además, este metal juega un rol protagónico en la regulación del apetito de manera similar a la leptina [34].

Por lo anterior, es muy importante conocer los valores de zinc presentes en el organismo con el propósito de prevenir enfermedades, hacer diagnósticos oportunos e instaurar tratamiento adecuado. Algunos autores como Florea y colaboradores [28] reportaron algunos valores de referencia en matrices biológicas como sangre, saliva, orina, cabello y uñas (tabla 2). 


\section{Plomo $(\mathrm{Pb})$}

El plomo es un metal flexible, inelástico y altamente tóxico para el ser humano. Su punto de fusión es de $328^{\circ} \mathrm{C}$ y su punto de ebullición de $1740{ }^{\circ} \mathrm{C}$; en ese sentido, es importante resaltar que, a partir de los $600{ }^{\circ} \mathrm{C}$, desprende abundantes vapores [35], entonces este metal puede ser inhalado en forma de vapores, polvo fino ambiental o humo.

La presencia de plomo en nuestro organismo se relaciona con contaminación ambiental, algunas de las fuentes de emisión y exposición a este elemento son: combustibles; restos de pinturas que se raspan y que pueden contaminar el suelo, lo cual es un peligro permanente, debido a que el plomo en el suelo no se degrada ni se puede retirar fácilmente; también, cañerías de agua cuyas tuberías o tanques incluyen plomo en su constitución [36], baterías, juguetes, artículos escolares, cerámicos, lacas, tintas, imprentas y diversas actividades industriales [37].

El ingreso de plomo a nuestro organismo se puede dar por vía aérea o por vía oral. Cuando llega por vía aérea, las partículas más grandes pueden quedar atrapadas en el tracto respiratorio, mientras las partículas pequeñas, es decir, menores a una micra, llegan directamente a los sacos alveolares de los pulmones, donde son absorbidas para dirigirse hacia la circulación sanguínea y son atacadas por el sistema linfático y los macrófagos alveolares; en ocasiones, estas partículas se pueden expulsar por expectoraciones.

La contaminación por vía oral se genera por el mal empleo de las reglas de higiene personal cuando se trabaja cerca del plomo, lo que ocasiona que se introduzca un gran porcentaje de este metal por ingesta y pueda viajar por el tracto gastrointestinal [35]. Existen algunos elementos y sustancias cuya disminución en la ingesta puede favorecer el aumento en la absorción de plomo, como hierro, calcio, fósforo, zinc y vitaminas como la E, C y Tiamina [35].

La exposición a plomo ocasiona algunas alteraciones a nivel hematológico como anemia microlítica, hipocrómica y la presencia de eritrocitos con punteado basófilo. Otras alteraciones incluyen a nivel gastrointestinal presencia de cólico saturnino; en el Sistema Nervioso Periférico provoca neuropatía periférica de predominio motor en los músculos de las extremidades, y alteraciones de la barrera neurocapilar con aumento en la presión del fluido endoneural, lo que genera compresión nerviosa, a su vez esto ocasiona isquemia, necrosis; degeneración axonal, desmielinizacion segmentaria y retarda la conducción nerviosa motora, con depresión del potencial de placa por bloqueo presináptico [38].

Por su parte, a nivel del Sistema Nervioso Central existe evidencia experimental que indica que niveles de plomo en sangre $>10 \mu \mathrm{g} / \mathrm{dL}$ pueden tener efectos perjudiciales y permanentes en la función cerebral, que incluyen reducción en la inteligencia, problemas de conducta y disminución del rendimiento escolar [39]; además, provoca inhibición de los canales de calcio, con la subsecuente disminución en la concentración intracelular, lo que altera la actividad neuronal y la secreción de neurotransmisores. En el adulto, los efectos biológicos por exposición inadecuada se caracterizan por un cuadro demencial, cefaleas, trastornos del sueño, trastornos de la visión, ataxia y trastornos del habla [35].

La medición de los niveles de este metal ha sido documentada por varios autores, quienes reportan algunos valores de referencia en distintas muestras biológicas (tabla 2).

\section{Cadmio (Cd)}

Este metal se encuentra en la tierra en cantidad aproximada a $0,1 \mathrm{ppm}$ y no se encuentra aislado de manera natural, ya que su disposición química como catión divalente hace que se encuentre asociado a otros compuestos [40]. El cadmio es utilizado ampliamente en la industria para la elaboración de pantallas de televisores, baterías, cigarrillos, soldaduras entre muchas otras funciones [40].

El cadmio puede estar en contacto con el ser humano por medio de una exposición directa o por ingesta de algunas comidas, por consumo de agua contaminada o por inhalación. Biológicamente, el cadmio no es de carácter esencial para el desarrollo de las funciones en el ser humano, pero puede ser tóxico para las células del organismo y acumularse en los tejidos con una vida media de 25 años. Algunos tejidos son muy susceptibles a este metal, como el hígado, los riñones, los testículos y los pulmones, aunque también se disemina en la sangre con una vida media de tres a cuatro meses [41]. Los niveles elevados de cadmio generan toxicidad capaz de inducir daño en los tejidos por producir 
alteraciones fisiológicas; su órgano blanco principal es el riñón y ocasiona defectos en varias proteínas, vitaminas y minerales, lo que podría derivar en osteoporosis; además, en el sistema cardiovascular puede ocasionar hipertensión arterial; otro de sus efectos podría ser la inhibición de la función normal del zinc y el magnesio en el cuerpo. A nivel endocrino, el cadmio produce cambios plasmáticos en los niveles de hormonas segregadas por la glándula pituitaria, alteración en la secreción de la hormona del crecimiento y la inhibición en la secreción de progesterona, algunos efectos son la reducción de peso en los recién nacidos, abortos espontáneos $y$ alteraciones en el desarrollo gonadal con deterioro del esperma [42].

Debido a la posibilidad de generar múltiples afecciones en el organismo, cuando los niveles de cadmio se elevan de forma excesiva, es necesario monitorear las concentraciones considerando diversas muestras biológicas, con el fin de prevenir la aparición de alteraciones sistémicas [43]. Los valores de referencia reportados suelen variar en virtud de la matriz biológica utilizada (tabla 2).

\section{Matrices biológicas para medición de metales pesados}

\section{Saliva}

La saliva es un fluido que tiene diversas funciones relacionadas con el mantenimiento de la homeostasis en el medio bucal, ayuda a la digestión mecánica de los alimentos por medio de la alfa-amilasa salival y otras enzimas para permitir la conformación del bolo alimenticio [44]; además, contribuye con el sistema inmunológico del organismo mediante una serie de proteínas específicas y no específicas [45].

La saliva tiene una composición que le otorga unas propiedades reológicas, que son las responsables de las diferencias entre los tipos de saliva serosa, mucosa o mixta. La cantidad de flujo salival varía por diversas condiciones fisiológicas en los individuos, tales como la edad, la presencia de alguna enfermedad, la ingesta de algunos medicamentos, el momento específico del día, entre otras, por lo que es necesario considerar que la secreción varía entre los individuos [46]. Este fluido bucal ha sido considerado una matriz biológica para la determinación de algunas sustancias en el cuerpo, lo cual ha incrementado el interés diagnóstico por medio del análisis salival, ya que como muestra permite la aplicación de un método seguro, simple y no invasivo para su recolección que beneficia al operador y al paciente; además, su almacenamiento es fácil y los costos son muy asequibles.

Estas características le confieren la posibilidad de ser utilizada para determinar y monitorear biomarcadores y ciertas sustancias químicas de interés particular, cuando otros fluidos como la sangre y la orina no se encuentran disponibles [47], [48]; sin embargo, algunos limitantes en esta muestra impiden que sea seleccionada como matriz por excelencia; entre estos es posible considerar que el flujo de iones en la saliva varia significativamente durante el transcurso del día y en la saliva pueden estar presentes otras sustancias, tales como restos de comida, bacterias y células epiteliales que pueden alterar las mediciones de algunas sustancias. También es posible que durante la recolección se contamine la saliva con otros fluidos como sangre o por la manipulación inadecuada durante el procedimiento; además, no existen protocolos ni materiales de referencia estándar o certificados ni valores de referencia fiables para la población humana [49].

A pesar de estas limitaciones, es preciso mencionar que en las últimas décadas el análisis salival ha tenido gran auge al ser utilizado en la determinación de abuso de drogas [50], [51], en el seguimiento a individuos fumadores y para determinar trazas de metales pesados [41]. Algunos de los metales que han sido aislados en saliva son: cadmio, plomo, zinc, mercurio, titanio, cromo, hierro y cobalto [46], [48], [52]; sin embargo, es necesario tener presente que en la saliva las concentraciones de estos compuestos metálicos son bajas [53].

Siempre que se utilice la saliva como matriz biológica es necesario indagar por la fiabilidad de los reportes que se pueden obtener y considerar el control de factores locales que puedan influirlos; por ejemplo, en caso de estimaciones de plomo y cadmio en saliva existe potencial confusión, ya que si están presentes restauraciones intraorales, puede existir liberación de trazas de estos metales; además, existe la probabilidad de contaminación no intencional [54]; sin embargo, para el caso del plomo, se ha sugerido que la concentración salival se relaciona con exposición reciente a este metal y por esto puede constituir un indicador útil y no 
invasivo para valorar el estado del metal en el organismo [55].

En el caso del mercurio, Zimmer [56] considera que los niveles en saliva no se correlacionan con las concentraciones en sangrey orina, pero sícon el número de amalgamas o de las superficies dentales restauradas, por lo que el monitoreo de mercurio no se recomienda en saliva [56].

La recolección de muestra salival implica tomar en cuenta algunas consideraciones generales como la hora del día en que se realiza y la higiene bucal, por lo que es recomendable que los sujetos de estudio no ingieran alimentos ni bebidas, ni realicen actividades como fumar y masticar gomas de mascar durante 30 minutos previos a la obtención de las muestras, con el fin de evitar la contaminación [52]. Para uso en ciencias de la salud, se comercializan diferentes dispositivos de toma de muestras de saliva, que consisten en un kit que contiene un tubo de ensayo y un tampón con un conservante que permite estabilizar las muestras y evita la degradación microbiológica [57]. Es relevante establecer las condiciones ambientales y del sujeto para la toma de la muestra, ya que la cantidad del fluido requerido variará dependiendo de los analitos que sean considerados; también el transporte y el almacenamiento serán diferentes según cada caso.

\section{Cabello}

El uso de cabello como muestra biológica para la determinación de la presencia de algunas trazas de elementos ha sido controversial y se remonta a 1960, cuando algunos científicos reactivaron el uso de cabello para valoraciones asociadas con nutrición; sin embargo, la posibilidad de utilizarlo para el análisis químico ha generado gran interés y motivación por demostrar en qué casos se puede utilizar y la importancia de conocer el comportamiento de las trazas de elementos para lograr unirlas al cabello, ya que muchas veces este mecanismo es desconocido.

El uso de cabello ha sido justificado por su fácil obtención y por reflejar información más antigua en comparación con la orina, la sangre y la saliva, por lo que resulta útil en caso de determinar la exposición crónica a cierta sustancia; además, el cabello es fácil de transportar y tiene buena estabilidad durante el almacenamiento a temperatura ambiente [58].
Algunas aplicaciones en las que se utiliza cabello como muestra son: monitoreo de exposiciones ambientales [59], control de abuso de drogas [60], [61], exposición a compuestos organofosforados, identificación de algunas trazas de elementos de interés biológico como aluminio, cobre, hierro, estroncio y zinc. Con respecto a este último metal $(\mathrm{Zn})$, la determinación en cabello puede informar del historial de ingesta; pero existe un inconveniente en la correlación de los niveles de zinc en cabello y zinc plasmático, ya que el zinc del cabello no se recambia [32]. En el cabello también es posible realizar determinación de elementos que pueden ser potencialmente dañinos, pues se consideran causantes de efectos cancerígenos como arsénico, cadmio, cromo, mercurio, níquel y plomo [62].

En caso de exposición a metales pesados, es necesario considerar que, debido a que el cabello crece aproximadamente $10 \mathrm{~mm}$ por mes, sirve como muestra para hacer un seguimiento a largo plazo de exposiciones pasadas y recientes, reflejando concentraciones medias de exposición en los meses anteriores [63]. Los niveles de metales presentes en el cabello pueden variar en virtud de algunos factores como la edad, el sexo, el color y el cuidado del cabello, el hábito de fumar y los factores étnicos [64].

La toma de muestras de cabello varía según los investigadores, por ejemplo, Blaurock plantea que se realiza cerca del cuero cabelludo de la zona occipital [65]. La longitud de las hebras a obtener oscila entre $4-5 \mathrm{~cm}$ y se procede al corte con tijeras de acero inoxidable previamente enjuagadas en etanol para remover los contaminantes externos [66]. Luego, las muestras requieren lavado en repetidas ocasiones utilizando un detergente desionizado y tres (3) enjuagues posteriores con agua desionizada [65]. El secado de las muestras se realiza a $60^{\circ} \mathrm{C}$ durante 4 horas en un horno eléctrico previamente designado [67]. Para la digestión de la muestra, se utilizan ácidos certificados como libres de metal [65]. Pese a esta breve descripción, existen diferencias en los reportes de la literatura, por lo que falta estandarización de procesos y protocolos para recolección de muestra, transporte y almacenamiento, así como procesamiento de cabello.

\section{Sangre}

La sangre es el fluido que circula por el sistema vascular; está compuesta por plasma, el cual incluye 
sustancias inorgánicas como agua, sales minerales o electrolitos y sustancias orgánicas como proteínas plasmáticas, sustancias nutritivas, gases, productos del metabolismo proteico, hormonas y anticuerpos; además, contiene grupos celulares como eritrocitos, leucocitos y plaquetas. La cantidad de sangre en el cuerpo humano varía en cada individuo dependiendo de la edad, el tipo corporal y el sexo; pese a esto se ha establecido que una persona adulta sana en promedio posee $71 \mathrm{ml} \mathrm{de}$ sangre por kilo de peso corporal $(71 \mathrm{ml} \times 70 \mathrm{~kg}=$ $4970 \mathrm{ml}$ o 5 litros) [68].

La sangre es una matriz biológica que se emplea con frecuencia para evaluar la exposición a plomo y cadmio. Entre las ventajas de su uso, se encuentra la posibilidad de evaluar intoxicaciones agudas debido a la eficiencia y sensibilidad, ya que nos ofrece la dosis promedio de estos metales que ha sido recibida durante las semanas inmediatamente anteriores al día en que se obtiene la muestra; también, posibilita obtener la cantidad suficiente de la muestra y repetición de las pruebas en caso de algún error [69]. En caso de intoxicación crónica por plomo, no es tan efectivo el uso de sangre, ya que no ofrece el valor real acumulado en el organismo a largo plazo [35].

La Scientific American ha definido una medida límite del valor de plomo; esta es el índice de exposición biológica conocido en inglés como BEIha, cuyo valor es de 30 microgramos/100 ml de sangre; las concentraciones superiores a 50 microgramos/100 $\mathrm{ml}$ de sangre se asocian con alteraciones clínicas, mientras que las intoxicaciones graves se han reportado con concentraciones superiores a 120 microgramos $/ 100 \mathrm{ml}$ de sangre [70].

Dependiendo de la medición que se vaya a realizar, la cantidad de sangre requerida como muestra variará. Se ha reportado que $30 \mathrm{ml}$ pueden ser suficientes [71]; además, el contenido de los tubos en los que se almacenará pueden o no contener anticoagulante; estos requerimientos serán diferentes según el objetivo del examen, por lo que resulta útil revisar los protocolos. Todas las muestras se deben identificar con su etiqueta; ese mismo día, se debe realizar la centrifugación, la preparación y el traslado hacia el laboratorio de referencia para que finalmente se proceda con las determinaciones analíticas. En todo el proceso es preciso no romper la cadena de frío, la conservación se recomienda a una temperatura de $-20^{\circ} \mathrm{C}$ [72].

\section{Orina}

La orina es un desecho metabólico que proviene del ultrafiltrado del plasma a nivel renal; se encuentra constituida por un $95 \%$ de agua y $5 \%$ de solutos, aunque puede haber variaciones considerables en las concentraciones de estos solutos debido a la influencia de diversos factores como el aporte dietético, la actividad física, el metabolismo corporal, las funciones endocrinas e incluso la posición del cuerpo, por lo que la orina ha sido utilizada para analizar biomarcadores de diferentes enfermedades [73]; además, el uso de esta matriz ofrece una cantidad disponible abundante, si se considera que un adulto puede expulsar un volumen promedio de 1200-1500 ml de orina en un periodo de 24 horas [74]. Los solutos que conforman la orina pueden ser orgánicos e inorgánicos; los primeros son principalmente la urea y la creatinina [75], aunque en procesos patológicos como la diabetes se puede hallar glucosa en muestras de orina. En tanto que los solutos inorgánicos son cloro, potasio, sodio y, en menor cantidad, se encuentran metales pesados [76] como zinc, cadmio, cobre, mercurio y plomo, de los cuales diversos autores han reportado algunos niveles de referencia (tabla 2 ).

La obtención de la muestra de aproximadamente $100 \mathrm{ml}$ se realiza en horas de la mañana utilizando un recipiente de polietileno estéril. Las muestras se almacenan a $-20^{\circ} \mathrm{C}$ hasta que se realiza el análisis. Para este último se descongelan a $4{ }^{\circ} \mathrm{C}$, se homogenizan y filtran, y se dividen en alícuotas, en tubos de polietileno pretratados con ácido nítrico y lavados con agua ultrapura [77].

\section{Uñas}

Las uñas están compuestas de queratina, la cual se une fuertemente al formar enlaces disulfuros junto con moléculas de cisteína, siendo estos los responsables de la alta durabilidad y resistencia de las fibras de las uñas [78]. Dichas estructuras carecen de actividad metabólica y, en términos generales, constituyen una matriz biológica cuya muestra es fácil de colectar, ya que no requiere de método invasivo y existe facilidad para el almacenamiento, por lo cual son útiles en caso de determinar exposición crónica a ciertos contaminantes. Una aplicación muy reportada es la identificación de cadmio en personas fumadoras y en aquellas que se dedican a realizar actividades de minería; 
así como cuando se van a identificar partículas de metales pesados de gran toxicidad como mercurio, cadmio y plomo en personas que han estado expuestas por un tiempo prolongado [78], [79]. En uñas también se han medido niveles de zinc, ya que ofrecen información sobre el historial de ingesta, pero se presenta un inconveniente en la correlación de los niveles de zinc en uñas y zinc plasmático, ya que, como se mencionó antes, las uñas carecen de actividad metabólica, entonces el zinc no se recambia [32].

Con respecto a la recolección de esta muestra, existe la posibilidad de que los mismos individuos corten las uñas utilizando un cortaúñas limpio, retirando los sucios visibles por medio de pinzas recubiertas con teflón. Después, personal capacitado se encarga de someter la muestra a tres lavados con detergente no iónico ( $1 \% \mathrm{v} / \mathrm{v}$ Triton X-100) y luego a tres enjuagues con agua desionizada. Luego del lavado, las muestras de uñas se someten a secado en un horno eléctrico a $60^{\circ} \mathrm{C}$ y luego la muestra se almacena en bolsas de polietileno [79]. Al igual que el uso de cabello y la saliva como muestras biológicas para la medición de metales pesados, existe falta de consenso entre los criterios de los autores para la estandarización de los procedimientos y protocolos (tabla 1).

\section{Algunos usos y aplicaciones de la medición de metales}

A continuación, y con el fin de presentar argumentos para justificar la medición de metales pesados en el organismo, se presentan solo algunas situaciones o ejemplos en los que puede ser útil (tabla 1).

La medición de metales, como se ha planteado, se puede realizar en diversas matrices biológicas. En lo que respecta a saliva y cabello, un estudio realizado por Bales [80] para la determinación de sedimentos de cobre en seres humanos demostró que el contenido del dicho metal en estas muestras se reduce significativamente con el avance de la edad, por lo que sería útil considerar este tipo de valoraciones para estudiar el proceso de envejecimiento [80]. Otra aplicación reportada es la medición de niveles de algunos metales pesados para determinar la presencia y el estado de algunas enfermedades; por ejemplo, al comparar individuos con y sin
Alzheimer, se han realizado mediciones del nivel de zinc en las uñas, con diferencias entre los valores de los dos grupos [81], siendo mayores para los individuos que presentan esta alteración.

En otros estudios, la concentración de algunos metales pesados ha sido relacionada con ciertos desórdenes de tipo neurológico, como es el caso de los desórdenes en el aprendizaje; tras comparar niños en edad escolar con y sin problemas de aprendizaje en virtud de los niveles de plomo, cadmio, mercurio, zinc y cobre en sangre, se presentan diferencias en los valores [82]. Otro trastorno neurológico que se ha valorado es el desarrollo del autismo, cuya etiología no es conocida con exactitud pero que se asocia con exposiciones ambientales. Se han realizado evaluaciones de los niveles de plomo y mercurio en cabello en grupos de niños que presentan y no presentan este trastorno, con el fin de encontrar evidencia acerca de la exposición y contribuir a esclarecer la etiología [83].

Existen otras situaciones en las que enfermedades de gran importancia a nivel mundial por su alta prevalencia y mortalidad han sido valoradas desde la perspectiva de las exposiciones ambientales a ciertos materiales o elementos como los metales pesados. Tal es el caso del cáncer, para lo cual se ha reportado que la presencia de cadmio en orina se comporta como factor de riesgo para cáncer de mama en mujeres con alta densidad mamográfica en edades premenopausicas entre los 40 y 45 años [84].

En arterosclerosis coronaria existen múltiples factores de riesgos considerados, entre los cuales se incluye la exposición a metales como el cadmio, el plomo y el mercurio; sin embargo, estos metales no han sido estudiados comúnmente en investigación cardiovascular [85], pero sí existen estudios en los que ya se han realizado algunas de estas mediciones. En ocasiones, el monitoreo de los niveles de metales es de gran utilidad cuando se requiere dar seguimiento a exposiciones ambientales en trabajadores del sector industrial, relacionándolos con algunos hábitos y su estilo de vida; los elementos evaluados suelen ser cadmio, cromo, manganeso, níquel y plomo en matrices biológicas como sangre, orina, vello axilar y saliva [63]. 
Tabla 1. Reporte de la medición de metales pesados en algunas matrices biológicas

\begin{tabular}{|c|c|c|c|}
\hline Datos del estudio & Muestra & $\begin{array}{l}\text { Valores de } \\
\text { referencia }\end{array}$ & Valores reportados \\
\hline $\begin{array}{l}\text { Wang, D. [86] } \\
2008 \text { China }\end{array}$ & $\begin{array}{l}82 \text { sujetos } \\
\text { - Casos: } 49 \text { trabajadores } \\
\text { de fábrica } \\
\text { - Control: } 33 \text { soldados que } \\
\text { residen cerca a las fábricas }\end{array}$ & NE & $\begin{array}{l}\text { Sa (Trabajadores: Cu: } 28.5 \pm 13.9 \mu \mathrm{g} / \mathrm{L} ; \mathrm{Zn}: 191 \pm 107 \mu \mathrm{g} / \mathrm{L} ; \mathrm{Cd}: \\
0.36 \pm 0.24 \mu \mathrm{g} / \mathrm{L} ; \mathrm{Pb}: 24.9 \pm 11.8 \mu \mathrm{g} / \mathrm{L} \\
\text { Soldados: Cu: } 19.6 \pm 13.6 \mu \mathrm{g} / \mathrm{L} ; \mathrm{Zn}: 260 \pm 132 \mu \mathrm{g} / \mathrm{L} ; \mathrm{Cd}: 0.43 \pm 0.43 \\
\mu \mathrm{g} / \mathrm{L} ; \mathrm{Pb}: 25.5 \pm 14.4 \mu \mathrm{g} / \mathrm{L} \text { ) } \\
\text { S (Trabajadores: Cu: } 970 \pm 308 \mu \mathrm{g} / ; \mathrm{Zn}: 1.05 \pm 0.15 \mathrm{mg} / \mathrm{L} ; \mathrm{Cd}: \\
0.40 \pm 0.46 \mu / \mathrm{L} ; \mathrm{Pb}: 19.5 \pm 14.4 \mu \mathrm{g} / \mathrm{L} \\
\text { Soldados: } \mathrm{Cu}: 822 \pm 159 \mu \mathrm{g} / \mathrm{L} ; \mathrm{Zn}: 1.18 \pm 0.214 \mathrm{mg} / \mathrm{L} ; \mathrm{Cd}: \\
0.60 \pm 0.42 \mu / \mathrm{L} ; \mathrm{Pb}: 28.3 \pm 15.6 \mu \mathrm{g} / \mathrm{L} \text { ) }\end{array}$ \\
\hline $\begin{array}{l}\text { González, H. [87] } \\
1997 \text { México }\end{array}$ & 100 sujetos & Sa $(4,8$ ug/100mL) & Sa (Pb: 3,1ug/dL; Cd: $0,25 \mu \mathrm{g} / \mathrm{dL})$ \\
\hline $\begin{array}{l}\text { Arai, F. [88] } \\
\text { 1994 Japón }\end{array}$ & $\begin{array}{l}132 \text { sujetos } \\
-70 \text { expuestos por trabajo } \\
-62 \text { residentes de la zona } \\
\text { cercana }\end{array}$ & NE & 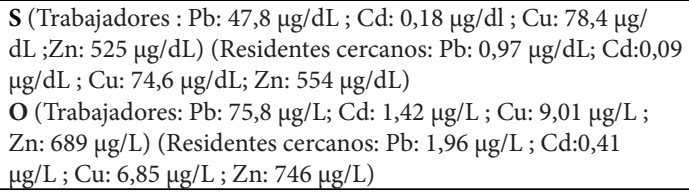 \\
\hline $\begin{array}{l}\text { Yllmaz, S. [89] } \\
2012 \text { Turquía }\end{array}$ & $\begin{array}{l}\text {-Agua } \\
\text {-comida }\end{array}$ & $\begin{array}{l}\mathrm{U}(\mathrm{Zn}: 73-191 \mu \mathrm{g} / \mathrm{g}) \\
\mathrm{Ca}(\mathrm{Zn}: 26-340 \\
\mu \mathrm{g} / \mathrm{g})\end{array}$ & $\mathbf{U}(\mathrm{Zn}: 155 \mu \mathrm{g} / \mathrm{g}) \mathbf{C a}(\mathrm{Zn}: 50 \mu \mathrm{g} / \mathrm{g})$ \\
\hline $\begin{array}{l}\text { Tëllez, J. } \\
\text { [38] } 2003 \text { Colombia }\end{array}$ & 7517 Trabajadores & $\begin{array}{l}\text { Sa (Pb: } 4,0 \\
\mu \mathrm{g} / 100 \mathrm{~mL})\end{array}$ & Sa ( Pb: 3,1ug/dL) \\
\hline $\begin{array}{l}\text { Jurczak, A. [90] } \\
2013 \text { Polonia }\end{array}$ & $\begin{array}{l}323 \text { mujeres } \\
-152 \text { usan terapia hormo- } \\
\text { nal para la menopausia } \\
-171 \text { no usan hormonas }\end{array}$ & $\begin{array}{l}\text { SS }(\mathrm{Zn}: 75-130 \\
\mu \mathrm{g} / \mathrm{dl}) \\
\text { S }(\mathrm{Pb}:<100 \mu \mathrm{g} / \mathrm{L} \\
\text { Cd: } 1.5 \mu \mathrm{g} / \mathrm{L})\end{array}$ & $\begin{array}{l}\text { SS (Grupo estudio, uso de hormonas: } \mathrm{Zn}: 0.76 \pm 0.21 \mathrm{mg} / \mathrm{L} \text { ) } \\
\text { (Grupo control: } \mathrm{Zn}: 0.68 \pm 0.15 \mathrm{mg} / \mathrm{L} \text { ) } \\
\text { S (Grupo estudio, uso de hormonas: } \mathrm{Pb}: 16.09 \pm 7.33 \mu \mathrm{g} / \mathrm{L} ; \mathrm{Cd} \text { : } \\
0.9 \pm 1.0 \mu \mathrm{g} / \mathrm{L} \text { ) (Grupo control: } \mathrm{Pb}: 20.18 \pm 9.01 \mu \mathrm{g} / \mathrm{L} ; \mathrm{Cd}: 0.8 \pm 1.1 \\
\mu \mathrm{g} / \mathrm{L} \text { ) }\end{array}$ \\
\hline $\begin{array}{l}\text { Parajuli, R. [91] } \\
2013 \text { Nepal } \\
\end{array}$ & $\begin{array}{l}-100 \text { mujeres embarazadas } \\
-100 \text { bebés }\end{array}$ & NE & $\mathbf{S}(\mathrm{Pb}: 31,7 \mu \mathrm{g} / \mathrm{L} ; \mathrm{Zn}: 2286 \mu \mathrm{g} / \mathrm{L})$ \\
\hline $\begin{array}{l}\text { Gil, F. [63] } \\
2010 \text { España }\end{array}$ & 178 trabajadores & NE & $\begin{array}{l}\text { S (Cd: } 0.45, \mu \mathrm{g} / \mathrm{L} ; \mathrm{Pb}: 5.26 \mu \mathrm{g} / \mathrm{L}) \\
\mathbf{O}(\mathrm{Cd}: 1.70, \mu \mathrm{g} / ; \mathrm{Pb}: 6.59 \mu \mathrm{g} / \mathrm{L}) \\
\mathrm{Ca}(\mathrm{Cd}: 0.23, \mu \mathrm{g} / \mathrm{L} ; \mathrm{Pb}: 0.75, \mu \mathrm{g} / \mathrm{L})\end{array}$ \\
\hline $\begin{array}{l}\text { Bjermo, H. }[92] \\
2012 \text { Suecia }\end{array}$ & 297 sujetos & NE & $\begin{array}{l}\text { S Pb:13.4 (5.8-28.6 } \mu \mathrm{g} / \mathrm{L}) ; \mathrm{Hg}: 1.13(0.31-3.45) \mu \mathrm{g} / \mathrm{L} ; \mathrm{Cd}: 0.19 \\
(0.09-1.08) \mu \mathrm{g} / \mathrm{L}\end{array}$ \\
\hline $\begin{array}{l}\text { Mortada, W. [79] } \\
2002 \text { Egipto }\end{array}$ & $\begin{array}{l}-68 \text { hombres } \\
-25 \text { mujeres }\end{array}$ & NE & $\begin{array}{l}\text { S (Cd: } 0.23 \mu \mathrm{g} / \mathrm{L} ; \mathrm{Hg}: 0.95 \mu \mathrm{g} / \mathrm{L} ; \mathrm{Pb}: 1.55 \mu \mathrm{g} / \mathrm{L}) \\
\text { O (Cd: } 0.32 \mu \mathrm{g} / \mathrm{L} ; \mathrm{Hg}: 0.89 \mu \mathrm{g} / \mathrm{L} ; 1.63 \mu \mathrm{g} / \mathrm{L}) \\
\text { Ca (Cd: } 0.015 \mu \mathrm{g} / \mathrm{L} ; \mathrm{Hg}: 0.037 \mu \mathrm{g} / \mathrm{L} ; \mathrm{Pb}: 0.065 \mu \mathrm{g} / \mathrm{L}) \\
\text { U ( Cd: } 0.018 \mu \mathrm{g} / \mathrm{L} ; \mathrm{Hg}: 0.033 \mu \mathrm{g} / \mathrm{L} ; \mathrm{Pb}: 0.062 \mu \mathrm{g} / \mathrm{L})\end{array}$ \\
\hline $\begin{array}{l}\text { Elhamri, H. [93] } \\
2007 \text { Marruecos }\end{array}$ & 108 sujetos & NE & Ca 0.22 a $9.56 \mu \mathrm{g} / \mathrm{g}$ \\
\hline $\begin{array}{l}\text { Díez, S. [94] } \\
2009 \text { España }\end{array}$ & 218 sujetos & $\begin{array}{l}0,1 \mu \mathrm{g} \mathrm{Hg} / \mathrm{kg} \text { de } \\
\text { peso corporal al día } \\
\text { (equivalente a } 1 \mu \mathrm{g} \\
\mathrm{Hg} / \mathrm{g} \text { de cabello) }\end{array}$ & $\begin{array}{l}\mathrm{V} / 2 \text { Consumo }>4 \text { veces } / \mathrm{Sem}: 1,40 \mu \mathrm{g} / \mathrm{g} \\
\mathrm{V} / 2 \mathrm{NO} \text { consumo: } 0,49 \mu \mathrm{g} / \mathrm{g} \\
\text { Niveles en } \mathrm{Ca} \text { en recién nacidos, ingesta materna }>2 \text { veces/se- } \\
\text { mana }=2,26 \mu \mathrm{g} / \mathrm{g} \\
\text { Niveles en Ca recién nacidos, ingesta materna }<2 \text { veces } / \text { semana }= \\
0,78 \mu \mathrm{g} / \mathrm{g}\end{array}$ \\
\hline $\begin{array}{l}\text { Hightower, JM. [26] } \\
2003 \text { EE.UU. }\end{array}$ & 123 sujetos & $\begin{array}{l}\mathbf{S}<5.0 \mu \mathrm{g} / \mathrm{L} \\
\mathbf{C a}<1.0 \mu \mathrm{g} / \mathrm{g}\end{array}$ & $\begin{array}{l}\text { S (Hg: } \geq 5.0 \mu \mathrm{g} / \mathrm{L}) \\
\mathrm{Ca}(\mathrm{Hg}: 1.55 \text { to } 14.81 \mu \mathrm{g} / \mathrm{g})\end{array}$ \\
\hline
\end{tabular}

Fuente: elaboración propia

\section{Conclusiones}

La cuantificación de metales pesados en muestras biológicas permite conocer posibles alteraciones bioquímicas generadas por variaciones en sus niveles.

Para la cuantificación de niveles de metales pesados en el organismo, se han tomado diferentes muestras biológicas teniendo en cuenta la sangre como matriz de referencia por considerar que refleja los valores más cercanos a la concentración real; sin embargo, se reporta como alternativa el uso de otras matrices biológicas como cabello, saliva, orina y uñas, cuyas ventajas son la recolección menos traumática en comparación con las muestras de sangre y la facilidad en el almacenamiento. El uso de matrices biológicas como saliva y sangre indican exposición reciente, mientras la 
orina y el cabello reflejan resultados de concentraciones por exposición crónica. Es necesario considerar que los niveles de metales pesados presentan variaciones para las distintas muestras biológicas, las cuales se pueden atribuir a factores locales (fisiológicos, nutricionales, presencia de hábitos nocivos) y ambientales (exposición directa e indirecta, contaminación propia del ambiente según su localización), por lo que se sugiere considerar todas las fuentes de exposición al momento de desarrollar mediciones con el fin de evitar sesgos en los resultados.

Tabla 2. Valores de referencia de algunos metales pesados (cadmio, mercurio, zinc, cobre, plomo)

\begin{tabular}{|l|l|l|l|l|l|}
\hline \multicolumn{1}{|c|}{ Metales/Muestras } & \multicolumn{1}{c|}{ Uñas } & \multicolumn{1}{c|}{ Cabello } & \multicolumn{1}{c|}{ Saliva } & \multicolumn{1}{c|}{ Orina } & \multicolumn{1}{c|}{ Sangre } \\
\hline Cadmio & $0,018 \mu \mathrm{g} / \mathrm{g}[95]$ & $0,41 \mu \mathrm{g} / \mathrm{g}[96]$ & $0,43 \mu \mathrm{g} / \mathrm{L}[97]$ & $0,32 \mathrm{~g} / \mathrm{L}[95]$ & $0,55 \mu \mathrm{g} / \mathrm{L}[51]$ \\
\hline Mercurio & $0,033 \mu \mathrm{g} / \mathrm{g}[95]$ & $0,037 \mu \mathrm{g} / \mathrm{g}[95]$ & $0,015 \mathrm{mg} / 100 \mathrm{ml} \mathrm{[102]}$ & $0,89 \mathrm{~g} / \mathrm{L}[95]$ & $0,95 \mathrm{~g} / \mathrm{L}[79]$ \\
\hline Plomo & $0,062 \mu \mathrm{g} / \mathrm{g}[95]$ & $4,06 \mu \mathrm{g} / \mathrm{g}[95]$ & $25,5 \mu \mathrm{g} / \mathrm{L} \mathrm{[96]}$ & $1,63 \mathrm{~g} / \mathrm{L}[95]$ & $56,8 \mathrm{mg} / \mathrm{L}[101]$ \\
\hline Zinc & $129-179 \mu \mathrm{g} / \mathrm{g}[28]$ & $102-258 \mu \mathrm{g} / \mathrm{g}[28]$ & $23-70 \mathrm{mg} / \mathrm{g}[28]$ & $0,5 \mathrm{mg} / \mathrm{g}[28]$ & $0,70-1,20 \mathrm{mg} / \mathrm{L}[97]$ \\
\hline Cobre & $65,8 \pm 17 \mathrm{ppm} \mathrm{[98]}$ & $5,0-55,0 \mu \mathrm{g} / \mathrm{g}[99]$ & $19,6 \mu \mathrm{g} / \mathrm{L} \mathrm{[96]}$ & $\begin{array}{l}11,38 \\
\mu \mathrm{g} / \mathrm{L}[100]\end{array}$ & $0,75-1,50 \mathrm{mg} / \mathrm{L}[97]$ \\
\hline
\end{tabular}

Unidades de medida: $\mathrm{g}$ (gramo); mg (miligramo); $\mu \mathrm{g}$ (microgramo); $\mathrm{ml}$ (mililitro); dl (decilitro); L (Litro).

Fuente: elaboración propia

\section{Referencias}

[1] Tabak LA. A Revolution in Biomedical Assessment: The Development of Salivary Diagnostics. J Dent Educ. 2001; 65:1335-9.

[2] Vardanyan LG, Ingole BS. Studies On Heavy Metal Accumulation in Aquatic Macrophytes from Sevan (Armenia) and Carambolim (India) Lake Systems. Environ Int. 2006;32(2):208-18.

[3] Ybañez N, Montoro R. Trace Element Food Toxicology: An Old and Ever-Growing Discipline. Crit Rev Food Sci Nutr. 1996;36(4):299-320.

[4] Behne D, Hammel C, Pfeifer H, Röthlein D, Gessner $\mathrm{H}$, Kyriakopoulos A. Speciation of Selenium in the Mammalian Organism. Analyst. 1998;123(5):871-3.

[5] Barregård L, Svalander C, Schütz A, Westberg G, Sällsten G, Blohmé I. Cadmium, Mercury, and Lead in Kidney Cortex of the General Swedish Population: A Study of Biopsies from Living Kidney Donors. Environ Health Perspect. 1999;107(11):867-71.

[6] Navarro J, Aguilar I, López J. Aspectos bioquímicos y genéticos de la tolerancia y acumulación de metales pesados en plantas. Ecosistemas. 2007;16(2):10-25.

[7] Järup L. Hazards of Heavy Metal Contamination. Br Med Bull. 2003;68:167-82.

[8] Nriagu JO. History of Global Metal Pollution. Science. 1996;272:223-4.

[9] Delgadillo A, González C, Priego F, Villagómez J, Acevedo O. Fitorremediación, una alternativa para eliminar la contaminación. Trop Subtrop Agro- ecosyt. 2011;14(2):597-612. Disponible en: http:// www.scielo.org.mx/scielo.php?script $=$ sci_arttext\&pid=S1870-04622011000200002\&lng=es.

[10] Stern BR, Solioz M, Krewski D, Aggett P, Aw TC, Baker S, et al. Copper and Human Health: Biochemistry, Genetics, and Strategies for Modeling Dose-Response Relationships. J Toxicol Environ Health B Crit Rev. 2007;10(3):157-222.

[11] Georgopoulos PG, Roy A, Yonone-Lioy MJ, Opiekun RE, Lioy PJ. Environmental Copper: its Dynamics and Human Exposure Issues. J Toxicol Environ Health B. 2001;4(4):341-94.

[12] Medeiros DM, Jennings D. Role of Copper in Mitochondrial Biogenesis Via Interaction with ATP Synthase and Cytochrome c Oxidase. J Bioenerg Biomembr. 2002;34(5):389-95.

[13] Keen CL, Clegg MS, Hanna LA, Lanoue L, Rogers JM, Daston GP. The Plausibility of Micronutrient Deficiencies Being a Significant Contributing Factor to the Occurrence of Pregnancy Complications. J Nutr. 2003;133(5 Suppl 2):1597S-1605S.

[14] Saari JT. Copper Deficiency and Cardiovascular Disease: Role of Peroxidation, Glycation, and Nitration. Can J Physiol Pharmacol. 2000;78(10):848-55.

[15] Uauy R, Olivares M, González M. Essentiality of Copper in Humans. Am J Clin Nutr 1998;67(5 suppl):952S-959S.

[16] Aydin A, Orhan H, Sayal A, Ozata M, Sahin G, Işimer A. Oxidative Stress and Nitric Oxide Related Parameters in Type II Diabetes Mellitus: Effects of Glycemic Control. Clin Biochem. 2001;34(1):65-70. 
[17] Flores L, Rodela S, Abian J, Clària J, Esmatjes E. F2 Isoprostane is Already Increased at the Onset of Type 1 Diabetes Mellitus: Effect of Glycemic Control. Metabolism. 2004;53(9):1118-20.

[18] Xu X, Pin S, Gathinji M, Fuchs R, Harris ZL. Aceruloplasminemia: An Inherited Neurodegenerative Disease with Impairment of Iron Homeostasis. Ann N Y Acad Sci. 2004;1012:299-305.

[19] Gaetke LM, Chow CK. Copper Toxicity, Oxidative Stress, and Antioxidant Nutrients. Toxicology. 2003;189(1-2):147-63.

[20] Hostynek JJ, Maibach HI. Copper Hypersensitivity: Dermatologic Aspects: An Overview. Rev Environ Health. 2003;18(3):153-83.

[21] Watanabe K, Tanaka T, Shigemi T, Hayashida Y, Maki K. Mn and Cu Concentrations in Mixed Saliva of Elementary School Children in Relation to Sex, Age, and Dental Caries. J Trace Elem Med Biol. 2009;23(2):93-9.

[22] Kao RT, Dault S, Pichay T. Understanding the Mercury Reduction Issue: The Impact of Mercury on the Environment and Human Health. J Calif Dent Assoc. 2004;32(7):574-9.

[23] Wolfe M, Schwarzbach S, Sulaiman R. Effects of Mercury on Wildlife: A Comprehensive Review. Environ Toxicol Chem. 1998;17(2):146-60.

[24] McDowell MA, Dillon CF, Osterloh J, Bolger PM, Pellizzari E, Fernando R. Hair Mercury Levels in U.S. Children and Women of Childbearing age: Reference Range Data from NHANEs 1999-2000. Environ Health Perspect. 2004;112(11):1165-71.

[25] Bates MN. Mercury Amalgam Dental Fillings: An Epidemiologic Assessment. Int J Hyg Environ Health. 2006;209(4):309-16.

[26] Hightower JM, Moore D. Mercury Levels in HighEnd Consumers of Fish. Environ Health Perspect. 2003;111(4):604-8.

[27] Homme KG, Kern JK, Haley BE, Geier DA, King PG, Sykes LK, Geier MR. New Science Challenges old Notion that Mercury Dental Amalgam is Safe. Biometals. 2014;27(1):19-24.

[28] Florea D, Molina J, Millán E, Sáez L, Pérez de la Cruz A, Planells P. Nosotros y el cinc. Nutr Hosp. 2012;27(3):691-700.

[29] Torres R, Bahr P. El zinc: la chispa de la vida. Rev Cubana Pediatr. 2004;76(4). Disponible en: http:// bvs.sld.cu/revistas/ped/vol76_4_04/ped08404.htm

[30] Hernández CM, Izquierdo A. Función del cinc en la recuperación inmunonutricional de lactantes malnutridos. Rev Cubana Aliment Nutr. 2000;14(1):6570.

[31] Salguerio M, Weill R, Hernández M, Zubillaga M, Lysionek A, Caro R. Deficiencia de zinc en relación con el desarrollo intelectual y sexual. Rev Cub Salud Pública. 2004;30(2). Disponible en: http://www.redalyc.org/pdf/214/21430207.pdf

[32] Roney N, Osier M, Paikoff SJ, Smith CV, Williams M, De Rosa CT. Agency for Toxic Substances and Disease Registry. ATSDR Evaluation of Potential for Human Exposure to Zinc. Toxicol Ind Health. 2007;23(5-6):247-308.

[33] Prasad AS. Recognition of Zinc-Deficiency Síndrome. Nutrition. 2001;17:67-9.

[34] Prasad AS. Clinical Manifestations of Zinc Deficiency. Annu Rev Nutr. 1985;5:341-63.

[35] Téllez J, Bautista F. Exposición ocupacional a plomo: aspectos toxicológicos. Avances en Enfermería. 2005;23(1):31-44.

[36] Peña C, Dean C, Ayala F. Toxicología ambiental: evaluación de riesgo y restauración ambiental. Arizona: The University of Arizona; 2001, p. 48-60.

[37] Fontana D, Lascano V, Solá N, Martínez S, Virgolini M, Mazzieri M. Intoxicación por plomo y su tratamiento farmacológico. Rev Sal Públ. 2013;(17)1:4959.

[38] Tellez J. "Metales pesados: mercurio, plomo y cromo en aspectos toxicologicos de plaguicidas y metales pesados”. En: Téllez J, Gutiérrez M, Patiño N. Memorias del curso taller aspectos toxicológicos de plaguicidas y metales pesados. Departamento de Toxicología, Facultad de Medicina, Universidad Nacional de Colombia, 2003.

[39] Lanphear BP, Hornung R, Khoury J, et al. Low-Level Environmental Lead Exposure and Children's Intellectual Function: An International Pooled Analysis. Environ Health Perspect. 2005;113(7):894-9.

[40] Bernhoft R. Cadmium Toxicity and Treatment. The Scien Wor J. 2013;2013. Disponible en: http://www. hindawi.com/journals/tswj/2013/394652/cta/

[41] Talio MC, Luconi MO, Masi AN, Fernández LP. Cadmium Monitoring in Saliva and Urine as Indicator of Smoking Addiction. Sci Total Environ. 2010;408(16):3125-32.

[42] Lafuente A. The Hypothalamic-pituitary-gonadal axis is Target of Cadmium Toxicity. An Update of Recent Studies and Potential Therapeutic Approaches. Food Chem Toxicol. 2013;59:395-404.

[43] Whanger PD. Cadmium Effects in Rats on Tissue Iron, Selenium, and Blood Pressure; Blood and Hair Cadmium in Some Oregon Residents. Environ Health Perspect. 1979;28:115-21.

[44] Amerongen AV, Veerman EC. Saliva-The Defender of the Oral Cavity. Oral Dis. 2002;8(1):12-22.

[45] Lawrence HP. Salivary Markers of Systemic Disease: Noninvasive Diagnosis of Disease and Monitoring of General Health. J Can Dent Assoc. 2002;68(3):170-4. 
[46] Chiappin S, Antonelli G, Gatti R, De Palo EF. Saliva Specimen: A New Laboratory Tool for Diagnostic and Basic Investigation. Clin Chim Acta. 2007;383(1-2):30-40.

[47] Lima DP, Diniz DG, Moimaz SA, Sumida DH, Okamoto AC. Saliva: Reflection of the Body. Int J Infect Dis. 2010;14(3):e184-8.

[48] Ben-Aryeh H, Sela J, Peled M. Salivary tests as a diagnostic tool in oral disease management. Refuat Hapeh Vehashinayim. 2002;19(3):51-90.

[49] Barbosa F Jr, Corrêa Rodrigues MH, Buzalaf MR, Krug FJ, Gerlach RF, Tanus-Santos JE. Evaluation of the Use of Salivary Lead Levels as a Surrogate of Blood Lead or Plasma Lead Levels in Lead Exposed Subjects. Arch Toxicol. 2006;80(10):633-7.

[50] Drummer OH. Drug testing in oral fluid. Clin Biochem Rev. 2006;27(3):147-159.

[51] Bosker WM, Huestis MA. Oral Fluid Testing for Drugs of abuse. Clin Chem. 2009;55(11):1910-31.

[52] Olmedo P, Pla A, Hernández AF, López-Guarnido O, Rodrigo L, Gil F. Validation of a Method to Quantify Chromium, Cadmium, Manganese, Nickel and Lead in human Whole Blood, Urine, Saliva and Hair Samples by Electrothermal Atomic Absorption Spectrometry. Anal Chim Acta. 2010;659(1-2):60-7.

[53] Esteban M, Castaño A. Non-invasive Matrices in Human Biomonitoring: A review. Environ Int. 2009;35(2):438-49.

[54] Pigatto PD, Arancio L, Guzzi G, et al. Metals From Amalgam in Saliva: Association with Lichenoid Lesions, Leukoplakia, Burning Mouth Syndrome. Toxicol Letters. 2005;158:SS169-S170.

[55] West C, Hardcastle J, Compton R. Sono-Electroanalytical Determination of Lead in Saliva. Electroanalysis. 2002;14(21):1470-8.

[56] Zimmer H, Ludwig H, Bader M, Bailer J, Eickholz P, Staehle HJ, Triebig G. Determination of Mercury in Blood, Urine and Saliva for the Biological Monitoring of an Exposure From Amalgam Fillings in a Group with Self-Reported Adverse Health Effects. Int J Hyg Environ Health. 2002;205(3):205-11.

[57] Langel K, Engblom C, Pehrsson A, Gunnar T, Ariniemi K, Lillsunde P. Drug Testing in Oral Fluid-Evaluation of Sample Collection Devices. J Anal Toxicol. 2008;32(6):393-401.

[58] Gjerde H, Øiestad E, Christophersen A. Using Biological Samples in Epidemiological Research on Drugs of Abuse. Norsk Epidemiologi. 2011;21(1):514.

[59] Varrica D, Tamburo E, Dongarrà G, Sposito F. Trace Elements in Scalp Hair of Children Chronically Exposed to Volcanic Activity (Mt. Etna, Italy). Sci Total Environ. 2014;470-471:117-26.
[60] Cairns T, Hill V, Schaffer M, Thistle W. Removing and Identifying Drug Contamination in the Analysis of Human Hair. Forensic Sci Int. 2004;145(23):97-108.

[61] Cirimele V, Kintz P, Dumestre V, Goullé JP, Ludes B. Identification of Ten Corticosteroids in Human Hair by Liquid Chromatography-Ionspray Mass Spectrometry. Forensic Sci Int. 2000;107(1-3):381-8.

[62] Chittleborough G, Steel BJ. Is Human Hair a Dosimeter for Endogenous Zinc and Other Trace Elements? Sci Total Environ. 1980;15(1):25-35

[63] Gil F, Hernández AF, Márquez C, et al. Biomonitorization of Cadmium, Chromium, Manganese, Nickel and Lead in Whole Blood, Urine, Axillary Hair and Saliva in an Occupationally Exposed Population. Sci Total Environ. 2011;409(6):1172-80. doi: 10.1016/j.scitotenv.2010.11.033. Epub 2011 Jan 6.

[64] Barbosa Jr F, Tanus-Santos JE, Gerlach RF, Parsons PJ. A Critical Review of Biomarkers Used for Monitoring Human Exposure to Lead: Advantages, Limitations and Future Needs. Environ Health Perspect. 2005;113(12):1669-74.

[65] Blaurock E, Amin OR, Rabah T. Heavy Metals and Trace Elements in Hair and Urine of a Sample of Arab Children with Autistic Spectrum Disorder. Maedica (Buchar). 2011;6(4):247-57.

[66] Mehra R, Juneja M. Elements in Scalp Hair and Nail Indicating Metal Body Burden in Polluted Environment. JSIR. 2005;64(2):119-24.

[67] Norom IC, Igwe JC, Ejimone JC. Multielement Analysis of Human Scalp Hair Samples from Three Distant Towns in Southeastern Nigeria. Afr J Biotechnol. 2005;4(10):1124-7.

[68] Carrillo J, Amador M. Maduración de las células sanguíneas. México D.F.: Editorial Cybercell; 2007.

[69] Mussali P, Tovar E, Valverde M, Rojas E. Biomarkers of Exposure for Assessing Environmental Metal Pollution: From Molecules to Ecosystems. Rev Int Contam Ambie. 2013;29(1):117-40.

[70] Kamal A, Qayyum M, Cheema I, Rashid A. Biological Monitoring of Blood Aphthalene Levels as a Marker of Occupational Exposure to PAHs among Auto-Mechanics and Spray Painters in Rawalpindi. BMC Public Health. 2011;11:467. Disponible en: http://www.biomedcentral.com/content/pdf/14712458-11-467.pdf

[71] García E, Pérez B, Fernández P, Fernández MA, De Paz C, Pérez AM, et al. Lead, Mercury and Cadmium in Umbilical Cord Blood and its Association with Parental Epidemiological Variables and Birth Factors. BMC Public Health. 2013;13:841. Disponible en: http://www.biomedcentral.com/1471-2458/13/841 
[72] Moreiras O, Cuadrado C. Ingesta de contaminantes (metales pesados) vía dieta total del Conjunto $\mathrm{Na}$ cional y Comunidades Autónomas: nuevo enfoque. Rev Clin Esp. 1993;193:76-81.

[73] King S, Schaub M. Análisis de orina y de los líquidos corporales. 5. ${ }^{\mathrm{a}}$ ed. Madrid: Editorial Médica Panamericana; 2010.

[74] Castaño A, Sánchez JE, Cañas A, Esteban M, Navarro C, Rodríguez AC. Mercury, Lead and Cadmium Levels in the Urine of 170 Spanish Adults: A Pilot Human Biomonitoring Study. Int J Hyg Environ Health. 2012;215(2):191-5.

[75] Schrier R. Diagnostic Value of Urinary Sodium, Chloride, Urea, and Flow. J Am Soc Nephrol. 2011;22(9):1610-3.

[76] Rahmoune H, Thompson PW, Ward JM, Smith CD, Hong G, Brown J. Glucose Transporters in Human Renal Proximal Tubular Cells Isolated from the Urine of Patients with Non-insulin-dependent diabetes. Diabetes. 2005;54(12):3427-34.

[77] Aguilera I, Daponte A, Gil F, Hernández AF, Godoy P, Pla A, et al. Urinary Levels of Arsenic and Heavy Metals in Children and Adolescents Living in The Industrialised Area of Ria of Huelva (sw Spain). Environ Int. 2010;36(6):563-9.

[78] Batista BL, Rodrigues JL, Nunes JA, Tormen L, Curtius AJ, Barbosa F Jr. Simultaneous Determination of $\mathrm{Cd}, \mathrm{Cu}, \mathrm{Mn}, \mathrm{Ni}, \mathrm{Pb}$ and $\mathrm{Zn}$ in Nail Samples by Inductively Coupled Plasma Mass Spectrometry (ICP-MS) after Tetramethylammonium Hydroxide Solubilization at Room Temperature: Comparison with ETAas. Talanta. 2008;76(3):575-9.

[79] Mortada WI, Sobh MA, el-Defrawy MM, Farahat SE. Reference Intervals of Cadmium, Lead, and Mercury in Blood, Urine, Hair, And Nails Among Residents in Mansoura City, Nile delta, Egypt. Environ Res. 2002;90(2):104-10.

[80] Bales CW, Freeland JH, Askey S, Behmardi F, Pobocik RS, Fickel JJ, et al. Zinc, Magnesium, Copper, and Protein Concentrations in Human Saliva: Age- and Sex-Related Differences. Am J Clin Nutr. 1990;51(3):462-9.

[81] Kuyumcu ME, Yesil Y, Ozturk ZA, Cankurtaran M, Ulger Z, Halil M, et al. An Alternative way for the Evaluation of Zinc Status in the Elderly; Nail Zinc Levels and Relationship with Alzheimer's Disease. Eur Rev Med Pharmacol Sci. 2013;17(11):1467-71.

[82] Yousef S, Eapen V, Zoubeidi T, Kosanovic M, Mabrouk AA, Adem A. Learning Disorder and Blood Concentration of Heavy Metals in the United Arab Emirates. Asian J Psychiatr. 2013;6(5):394-400.

[83] Lakshmi Priya MD, Geetha A. Level of Trace Elements (Copper, Zinc, Magnesium and Selenium) and Toxic Elements (Lead and Mercury) in the Hair and Nail of Children with Autism. Biol Trace Elem Res. 2011;142(2):148-58.

[84] Adams SV, Newcomb PA, Shafer MM, Atkinson C, Bowles EJ, Newton KM, et al. Urinary Cadmium and Mammographic Density in Premenopausal Women. Breast Cancer Res Treat. 2011;128(3):83744.

[85] Sponder M, Fritzer-Szekeres M, Marculescu R, Mittlböck M, Uhl M, Köhler-Vallant B, Strametz-Juranek J. Blood and Urine Levels of Heavy Metal Pollutants in Female and Male Patients with Coronary Artery Disease. Vasc Health Risk Manag. 2014;10:311-7. doi: 10.2147/VHRM.S61510. eCollection 2014.

[86] Wang D, Du X, Zheng W. Alteration of Saliva and Serum Concentrations of Manganese, Copper, Zinc, Cadmium and Lead Among Career Welders. Toxicol Lett. 2008;176(1):40-7.

[87] Banderas JA, Zacarías IG, Garduño R, Aceves E, González M. Electrophoretic Analysis of Whole Saliva and Prevalence of Dental Caries. A Study in Mexican Dental Students. Arch Med Res. 2002;33(5):499-505.

[88] Arai F, Yamamura Y, Yoshida M, Kishimoto T. Blood and Urinary Levels of metals $(\mathrm{Pb}, \mathrm{Cr}, \mathrm{Cd}, \mathrm{Mn}, \mathrm{Sb}$, $\mathrm{Co}$ and $\mathrm{Cu})$ in Cloisonne Workers. Ind Health. 1994;32(2):67-78.

[89] Basta N, Gradwohl R. Estimation of $\mathrm{Cd}, \mathrm{Pb}$, and Zn Bioavailability in Smelter-contaminated Soils by a Sequential Extraction Procedure. Journal of Soil Contamination. 2000;9(2):149-164.

[90] Jurczak A, Brodowski J, Grochans E, Karakiewicz B, Szkup-Jabłońska M, Wieder-Huszla S, et al. Effect of Menopausal Hormone Therapy on the Levels of Magnesium, Zinc, Lead and Cadmium in Post-Menopausal Women. Ann Agric Environ Med. 2013;20(1):147-51.

[91] Parajuli RP, Fujiwara T, Umezaki M, Watanabe C. Association of Cord Blood Levels of Lead, Arsenic, and Zinc with Neurodevelopmental Indicators in Newborns: A Birth Cohort Study in Chitwan Valley, Nepal. Environ Res. 2013;121:45-51.

[92] Bjermo H, Sand S, Nälsén C, Lundh T, Enghardt Barbieri H, Pearson M. Lead, Mercury, and Cadmium in Blood and their Relation to Diet among Swedish Adults. Food Chem Toxicol. 2013;57:161-9.

[93] Elhamri H, Idrissi L, Coquery M, Azemard S, El Abidi A, Benlemlih M, et al. Hair Mercury Levels in Relation To Fish Consumption in a Community of the Moroccan Mediterranean Coast. Food Addit Contam. 2007;24(11):1236-46.

[94] Díez S, Delgado S, Aguilera I, Astray J, Pérez-Gómez B, Torrent M, et al. Prenatal and Early Childhood 
Exposure to Mercury and Methylmercury in Spain, a High-fish-consumer Country. Arch Environ Contam Toxicol. 2009;56(3):615-22.

[95] Kaniewski A, Takagi Y, Marcinkowski JT. Concentrations of 21 Elements in Hair of People Living in Poznań. Probl Med Społ. 1997;32:391.

[96] Wang D, Du X, Zheng W. Alteration of Saliva and Serum Concentrations of Manganese, Copper, Zinc, Cadmium and Lead Among Career Welders. Toxicology Letters. 2008;176(1):40-7.

[97] Całkosiński I, Gamian A, Kobierska-Brzoza J, Fita K, Czajczyńska-Waszkiewicz A, Majda J. The Influence of Variable Environmental Factors on $\mathrm{Hu}$ man's Organism Adaptive Ability. Adv Clin Exp Med. 2009;18 (5):519-527.

[98] Sukumar A, Subramanian R. Relative Element Levels in the Paired Samples of Scalp Hair and Fingernails of Patients from New Delhi. Sci Total Environ. 2007;372(2-3):474-9.
[99] Radomska K, Graczyk A, Konarski J, Adamowicz B. Evaluation of macro- and micro Element Content in the Human Body Determined by Hair Analysis. Pol Tyg Lek. 1991;46:24.

[100] Aguilera I, Daponte A, Gil F, Hernández AF, Godoy P, Pla A, Ramos JL; Dasahu Group. Urinary Levels of Arsenic and Heavy Metals in Children and Adolescents Living in the Industrialised Area of Ria of Huelva (sw Spain). Environ Int. 2010;36(6):563-9.

[101]Cañas AI, Cervantes M, Esteban M, Ruiz-Moraga M, Pérez-Gómez B, Mayor J, et al. Blood Lead Levels in a Representative Sample of the Spanish Adult Population: The Bioambient.ES project. Int J Hyg Environ Health. 2014;217(4-5):452-9.

[102] Sepúlveda L, Agudelo L, Arengas A. El mercurio, sus implicaciones en la salud y en el ambiente. Revista Luna Azul. 1997;4. Disponible en: http://lunazul. ucaldas.edu.co/downloads/e4822404Revista4_8. pdf 